\title{
Trypanosoma cruzi Interaction with its Vectors and Vertebrate Hosts
}

\author{
Mario Steindel \\ Departamento de Microbiologia e Parasitologia, CCB, Universidade Federal de Santa Catarina, Caixa Postal 476, \\ 88040-900 Florianópolis, SC, Brasil
}

Key words: Trypanosoma cruzi - interaction - vectors - vertebrate hosts

Four very interesting papers were presented and discussed in this round table. Two of them presented results concerning the interaction of the parasite with the triatomine bug Rhodnius prolixus focusing both biological and "immunological" factors involved in this interaction. The other two, discussed the characterization of triatomine populations using different approaches such as biology, ecology, genetics and molecular methods.

Azambuja et al. presenting the "Immunity of Rhodnius prolixus: trypanosomatid-vector interactions" in this meeting, shows that Trypanosoma cruzi development in the triatomine vector gut may be affected by both vector and parasite intrinsic factors. Several authors have demonstrated the presence of different lectins in the gut as well as in the hemolymph in a variety of triatomine species. Studies with the T. cruzi Dm28c and Y strains revealed that these strains were unable to develop in the hemocoel of the vector as well as in the presence of the hemolymph in vitro. Under natural conditions, when $T$. cruzi infection occurs by the ingestion of bloodstream trypomastigotes from the vertebrate host during the feeding process, its development in the vector is restricted to the gut. Transformation from trypomastigote to epimastigote form takes place in the vector crop. Digestive proteases and hemolytic factors present in the crop and involved in the digestion process may be acting as a barrier for parasite development. It is also known that lectins present in the triatomine gut induces epimastigote agglutination, probably playing an important role on the development and maintenance of vector infection. It has been demonstrated that the agglutination of epimastigote forms by the crop lectin of $R$. prolixus is inhibited by sugars linked to p-nitrophenol such as pnitrophenil- $\alpha$-D-galactopyranoside.

Fax: +55-48-331.9258. E-mail: ccblmst@ccb.ufsc.br Received 9 June 1999

Accepted 9 August 1999
Recent studies revealed that both humoral and cellular responses of the vector may be involved on the parasite clearance. A strong agglutination activity against culture epimastigotes of $T$. cruzi strains from both sylvatic and domestic origins has been demonstrate in the hemolymph of Panstrongylus megistus, Triatoma infestans and $R$. prolixus (Lemos 1993). In this same study, T. cruzi epimastigote agglutination by hemolymph was inhibited only by galactose, or by other sugars presenting galactose in their composition. Trypomastigotes derived from tissue culture or from infected mice blood showed no agglutination with this hemolymph lectin. This specific galactose-bound lectin from the $R$. prolixus hemolymph presented in a SDS-PAGE gel a molecular weight of $40 \mathrm{kDA}$. A similiar molecular weight lectin was isolated from P. megistus hemolymph (Lemos 1993).

Differently from $T$. cruzi, $T$. rangeli develops in both triatomine gut and hemocoel, being recognized by the vector immune system. Experimental studies on $T$. rangeli infection in $R$. prolixus revealed the absence of effects in both erythrocyte or trypanolytic activity patterns observed in a noninfected insect hemolymph. However, a significant change on lysozyme levels, hemocyte number and prophenoloxidase (PO) activity were observed. Different immune responses could be detected in $R$. prolixus when the insects were inoculated with short or long epimastigote form of $T$. rangeli. When short epimastigotes were inoculated directly in the hemocoel, a high PO activity was detected in the hemolymph as well as in fat bodies, when the parasite rapidly disappeared. On the other hand, long epimastigotes develop well in the hemolymph. Only long epimastigotes were also found by our group in four different species of Rhodnius inoculated with $T$. rangeli strains isolated from different geographical regions. Protease activities in the hemolymph of $R$. prolixus were detected only in insects inoculated in the hemolymph with short epimastigotes. Protease activity was not detected neither in the hemolymph nor in the fat bodies when insects were fed with either short or long 
epimastigotes. Therefore, immune reactions induced by the presence of short and/or long epimastigotes may be responsible for differences in the parasite establishment and development in the hemolymph of $R$. prolixus.

During the last years, our group have been shown that $T$. rangeli is a polymorphic trypanosome species. Based on biological, immunological, biochemical and molecular methods, we have found that $T$. rangeli strains isolated from distinct geographical regions presents particular genetic characteristics (Steindel et al. 1994, Grisard et al. 1999). Moreover, a great adaptability of these parasites to the original local vectors was observed (Steindel \& Guarneri 1996, Grisard et al. 1999). For that, we consider that such refined analyses should consider as much different strains and vectors as possible to avoid wrong inferences.

Eloi S Garcia et al. discuss in their paper the role of neuroendocrine hormones in the maintenance of T. cruzi infection and metacyclogenesis in the triatomine vector. The infection and metacyclogenesis of different $T$. cruzi strains are related to both nature of parasite and susceptibilitiy of the triatomine species. The establishment of $T$. cruzi infection in the vector gut may be affected by several factors as crop lytic factors and the presence of lectins. In vitro assays revealed that metacyclogenesis may be induced by $\alpha^{\mathrm{D}}$-globin components present in the host blood.

It is well known that azadirachtin, an insect growth inhibitor compound, blocks the neuroendocrine activity affecting the production of the prothoracicotropic hormone (PTTH) in R. prolixus. These hypothesis was confirmed using the head transplant technique developed by this group. Recently, it was shown that azadirachtin treatment causes drastic changes in the epithelial cells of $R$. prolixus midgut. This effect was partially reverted by head transplantation from normal insects and ecdysone therapy. Decaptation as well as azadirachtin treatment induces a decrease of the $T$. cruzi infection in $R$. prolixus. Interestingly, head transplantation and ecdysone therapy reestablish the ability of $T$. cruzi to develop in both headless and azadirachtin treated insects. These results suggests that PTTH may act as an important factor on the midgut cell organization as well as in the establishment and maintenance of $T$. cruzi infection in $R$. prolixus.

Jean P Dujardin et al. discuss the idea of the triatominae as model of morphological plasticity under ecological pressure focusing that morphological differences may appear before significant genetic changes can be demonstrated. One clear example is the adaptation to more stable habitats producing faster and significant simplification of some characteres in Triatominae populations such as sensilla complexity, bilateral symmetry relation, body size reduction and sexual dimorphism in the transition from sylvatic to domestic habitat. It is suggested that morphological species of Triatominae should be treated as "ecological species". On the other hand, ecological factors may be driving to speciation in Triatominae. Minor morphological changes observed among these insects may occur in a faster manner than those consisting reproductive barriers. These morphological species of triatomine bugs would be "ecological species", a vision which fits with the concept of "evolutionary units". As an example, the closelyrelated species by genetic markers such as $T$. infestans, T. platensis and T. delpontei species group that presents morphological and ecological differences among themselves can be used. In accordance with data based on biological, morphological, isoenzymatic and cytogenetic studies, the genetic distance between T. platensis and $T$. delpontei, two morphological and ecological similar species, was grater than the distance obtained between $T$. platensis and T. infestans.

Fernando A Monteiro et al. used a molecular approach (mithocondrial DNA analysis), in the study of T. infestans and T. melanosoma populations. Besides isoenzyme profiles, cytogenetic and mithocondrial DNA analysis showed no significant differences between these two triatomine species. Infertility and morphometric differences observed between $T$. infestans and T. melanosoma were at the same level as those found between populations of $T$. infestans that co-exists in a same geographical region.

Phylogenetic analysis of $T$. infestans populations allowed the authors to observe differences from insects isolated in Argentina and Brazil from those isolated in Bolivia. Analysis of DNA sequences obtained from T. melanosoma isolated at northeast Argentina and T. infestans from the State of Paraná, Brazil, showed that these sequences were identical to each other, being very similar to other DNA sequences of T. infestans captured in other areas in Brazil and Argentina. However, these results are based on a single or few number of insects. Therefore, it is highly recommended to use as many individuals as possible in these analysis in order to make phylogenetic inferences.

\section{REFERENCES}

Grisard EC, Steindel M, Guarneri AA, Eger-Mangrich I, Campbell DA, Romanha AJ 1999. Characterization for Trypanosoma rangeli strains isolated in Central and South America: an overview. Mem Inst Oswaldo Cruz 94: 203-209.

Lemos EM 1993. Caracterização de Lectinas da Hemolinfa de Panstrongylus megistus com Atividade 
Aglutinante para Trypanosoma cruzi, Msc Thesis, Universidade Federal de Minas Gerais, Belo Horizonte, $73 \mathrm{pp}$.

Steindel M, Guarneri AA 1996. Interaction of trypanosomes with different triatomine species. Mem Inst Oswaldo Cruz 91 (Suppl.): 32-33.
Steindel M, Dias Neto E, Ribeiro-Rodrigues R, Carvalho Pinto CJ, Grisard EC, Menezes CLP, Murta SMF, Simpson AJG, Romanha AJ 1994. Randomly amplified polymorphic DNA (RAPD) and isoenzyme analysis of Trypanosoma rangeli strains. J Euk Microbiol 41: 261-267. 